\title{
LOS AUXILIARES HAVE Y BE EN LOS TIEMPOS ANALÍTICOS DEL INGLÉS
}

\author{
ANA ISABEL OJEA LÓPEZ \\ Universidad de Oviedo
}

\section{RESUMEN}

Este artículo se centra en las diferencias entre los auxiliares have y be en las formas analíticas del inglés. Defendemos que, mientras que el verbo to be en un auxiliar de aspecto gramatical, el verbo to have debe considerarse un auxiliar de tiempo. Esta diferencia explicará no sólo la contribución de cada uno de ellos a la temporalidad final de la proposición, sino también su relación e interacción con otros constituyentes de la oración (adverbiales, verbos modales, etc.).

Palabras clave: Lingüística, Sintaxis, Aspecto Gramatical.

\begin{abstract}
This paper offers an account of the differences between the auxiliaries have and $b e$ in perfect and progressive forms. We argue that whereas $b e$ is an aspectual auxiliary, have should better be treated as a temporal marker. This different role explains their contribution to the overall temporality of the proposition, but also their relationship and interaction with other constituents of the sentence (adverbials, modal verbs etc.).
\end{abstract}

Key words: Linguistics, Syntax, Grammatical Aspect.

1. Tiempo y aspecto. Es suficiente un somero repaso a alguna de las gramáticas descriptivas del inglés para comprobar que el tratamiento gramatical de las formas have y be en los tiempos analíticos de esta lengua suele ser equivalente: ambos son analizados como auxiliares aspectuales y muchas veces como opciones binarias, i.e. la manifestación léxica del rasgo [ \pm perfectivo]) en el paradigma de la clasificación verbal. ${ }^{1}$ Como ya hemos discu-

\footnotetext{
${ }^{1}$ Como ejemplo de estos enfoques, a veces incluso contradictorios, podríamos señalar el de Quirk et al. (1985: 181), que defienden que el aspecto puede ser perfectivo o imperfectivo en las formas compuestas, frente a lo que ocurre en las formas simples donde "a verb phrase is totally unmarked for aspect". Señalan
} 
tido en otros trabajos (cf. Ojea, 2003), nosotros defendemos que si bien es cierto que be es un auxiliar de aspecto, no puede decirse otro tanto de have, o, al menos, no sin matizar mucho cuál es la información aspectual que have añade en los tiempos compuestos. Abundaremos aquí en esta idea, comparando primero el papel de los dos auxiliares en la caracterización del aspecto gramatical en inglés, para después contrastar su funcionamiento entre sí y con otras formas que contribuyen a la expresión de temporalidad de la oración.

No hay duda de que los conceptos de tiempo y de aspecto están interrelacionados, hasta el punto de que es imposible tratarlos aisladamente. De hecho, no es del todo exacto definir el tiempo gramatical como una categoría deíctica que sitúa el acontecimiento verbal con respecto al momento del habla, dado que lo que realmente se ordena en el eje temporal es la parte de ese acontecimiento que le interesa resaltar al hablante (i.e. el inicio, la totalidad, sus fases internas de desarrollo...), y esto es precisamente lo que señala el aspecto verbal: tiempo y aspecto, pues, claramente interaccionan. Parafraseando a Smith (1991), el aspecto es como la lente de una cámara, y sólo lo que enfocamos con esa lente podremos situarlo con respecto al momento en que tiene lugar la emisión.

Por otra parte, la noción de aspecto tampoco es unitaria ya que, como es bien sabido, puede expresarse léxica y gramaticalmente. En el primer caso, hablamos de aspecto léxico, un concepto que ha estado presente en la tradición gramatical probablemente ya desde la distinción aristotélica entre verbos de kinesis y de energeia. Entre los rasgos que comúnmente se aceptan para clasificar los predicados verbales en este sentido tendríamos fundamentalmente tres: télico, dinámico y durativo. El primero alude al fin inherente que está implícito en la significación de algunos predicados, el rasgo [+dinámico] caracteriza a aquellos acontecimientos cuyo desarrollo implica fases diferenciadas y el [+durativo] a los que tienen duración interna. Entorno a ellos se puede materializar la clasificación aspectual de Vendler (1967):
1. a. Estados:
[-télico], [-dinámico], [+durativo]
b. Actividades:
[-télico], [+dinámico], [+durativo]
c. Realizaciones:
d. Logros:
[+télico], [+dinámico], [+durativo]
[+télico], [+dinámico], [-durativo]

Ahora bien, estos rasgos pueden modificarse en función de la estructura sintáctica en que se sitúe el evento, con interesantes consecuencias para la aspectualidad (y, consecuentemente, la temporalidad) final del verbo. Así, por ejemplo, el verbo write se define como [-télico, +dinámico, +durativo], (es decir, como una actividad), pero write a poem como [+télico, +dinámico, +durativo], o sea como una realización, mientras que write poems sigue siendo [-télico, +dinámico, +durativo]. O el verbo resemble tendría los rasgos [télico, -dinámico, +durativo], es decir, sería un estado, pero resemble more each day sería

\footnotetext{
también que el aspecto "complete (perfective) and incomplete i.e. in progress (imperfective) may combine in a single VP: I have been reading is both perfective and progressive" (Quirk et al. 1985: 189). Tratamientos semejantes se encuentran en otras gramáticas de referencia como Huddleston (1984), Aarts (1997), Napoli (1993), Radford (1997)... Por contra, Jespersen (1924) o Comrie (1976), entre otros, ya cuestionan que la aportación de have en las formas perifrásticas tenga que ver directamente con el aspecto gramatical.
} 
[+télico, +dinámico, -durativo], clasificable, por tanto, como logro. El aspecto léxico depende, pues, no sólo del significado inherente al verbo sino también de los argumentos y adjuntos verbales que acompañen a éste.

La aspectualidad final de un sintagma verbal dependerá también de cómo se quiera presentar el evento: en su totalidad, en su desarrollo interno, marcando su punto de inicio, o su punto final, como único, como repetido... Las lenguas poseen mecanismos morfológicos (i.e. prefijos y sufijos) y analíticos para conseguir estos efectos, y esto es lo que tradicionalmente conocemos como aspecto gramatical. Dado que este trabajo se limita a la comparación de have y be en las formas verbales compuestas, sólo nos referiremos aquí a las dos manifestaciones de aspecto gramatical relevantes para la argumentación: el aspecto [ \pm perfectivo]. El aspecto gramatical perfectivo "enfoca" la totalidad del acontecimiento verbal, de ahí que se sigan principalmente lecturas de tipo inceptivo, puntual o completivo; en cuanto al imperfectivo, no marca los límites del evento, y con él el acontecimiento verbal se presenta como habitual, iterativo o en desarrollo.

Señalábamos más arriba que la categoría tiempo no ordena directamente el sintagma verbal con respecto al momento del habla, sino que la relación entre ambos está mediada por el aspecto. Defendemos, pues, una naturaleza composicional de la temporalidad: un evento, con unos determinados rasgos aspectuales léxicos, se inserta en una estructura sintáctica, por lo que alguno de estos rasgos se pueden modificar; el hablante decide también qué parte del acontecimiento verbal quiere situar en el tiempo (i.e. aspecto gramatical), lo cual puede llevar a su vez a una modificación de los rasgos aspectuales léxicos. Por seguir con el ejemplo que utilizamos anteriormente, el verbo write [-télico, +dinámico, +durativo] se puede proyectar en la sintaxis con su argumento interno realizado como un SDet específico (write a letter), resultando entonces un Sintagma Verbal con los rasgos aspectuales [+télico, +dinámico, +durativo]. Si situamos este SV en su totalidad (i.e. marcando sus límites) en el eje temporal como anterior al momento del habla, es decir, si la forma es pasada y perfectiva, la implicación es que el telos del evento se ha alcanzado, tal y como se comprueba en (2); pero si lo que quiere situarse en el tiempo pasado es el desarrollo interno del acontecimiento y no éste en su totalidad (aspecto gramatical imperfectivo), la telicidad del predicado queda en suspenso, pudiendo incluso entenderse como cancelada en determinados contextos, de ahí la ambigüedad de (3): ${ }^{2}$

2. He wrote a letter

3. He was writing a letter

a) for two hours. Implicación: terminó en esas dos horas

b) when those men broke in. No sabemos si la actividad alcanzó su telos, i.e. si finalmente terminó de escribir la carta

Los ejemplos (2) y (3) también dan cuenta de un hecho significativo: todas las formas flexivas del verbo, sean simples o compuestas, marcan tiempo y aspecto gramatical. Es decir, no es cierto, como parece desprenderse de algunas gramáticas descriptivas y/o pedagógicas del inglés, que el aspecto gramatical tenga que ser analítico en esta lengua (vid.

\footnotetext{
${ }^{2}$ Esta es la esencia de la denominada "paradoja del imperfectivo".
} 
nota (1)). De hecho, las dos formas simples del paradigma verbal, el Presente Simple y el Pretérito, informan de aspecto [-perfectivo] y [+perfectivo] respectivamente, de ahí las lecturas de habitual e iterativo asociadas con el primero, y de completivo con el segundo. ${ }^{3}$ Los verbos auxiliares have y be añaden, como veremos, matices de tiempo o de aspecto, pero no son, pues, específicamente marcadores de aspecto gramatical.

2. Los auxiliares have y be. Los auxiliares de las formas temporales analíticas tienen una función común: ambos marcan el tiempo de referencia con respecto al cual se sitúa el evento verbal. Sin embargo su aportación concreta a la semántica del sintagma verbal es distinta. Comenzando con la perífrasis be+participio presente, la caracterización global de su temporalidad podría ser la siguiente:

4. $[$ be $<$ tiempo gramatical $><$ progresión $>]+$ verbo - ing $<$ imperfectivo $>$

Es decir, las denominadas formas progresivas o continuas son formas imperfectivas a las que el auxiliar añade un rasgo de desarrollo en progresión que las hace incompatibles con sintagmas verbales [-dinámicos], i.e. los que se entienden como un continuum sin fases de desarrollo interno: ${ }^{4}$

5. *John was resembling his father <-télico, -dinámico, +durativo>

6. *The box was containing a present <-télico, -dinámico, +durativo>

Tampoco, y por razones interpretativas similares, es posible obtener esta lectura de desarrollo interno cuando to be se combina con sintagmas verbales [-durativos], es decir, con logros; en estos casos el progresivo implica o bien repetición del evento verbal (7a,b), o que el telos no se ha alcanzado aún pero está en proceso de lograrse (7c):

7. a. That horse is jumping well

b. The President was nodding

c. He was dying

Finalmente, y dado su carácter imperfectivo (i.e. que no marca límites), las formas progresivas, tanto en presente como en pasado, pueden proyectar su significación hacia el futuro: ${ }^{5}$

8. The train is leaving in two hours

They said that the train was leaving in two hours

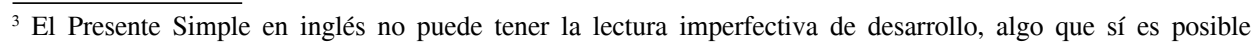
en otras lenguas como el español (Ej. ¿Qué hace el niño? Escribe la carta a los Reyes Magos).

${ }^{4}$ Efectivamente, el auxiliar progresivo be es incompatible con los predicados [-dinámicos], pero eso no implica que estos predicados no puedan presentarse como aspectualmente imperfectivos: ej. Knowing English, you can get a better job

${ }^{5}$ De nuevo encontramos aquí un interesante contraste con el español, donde sólo las formas imperfectivas simples, no las analíticas, permiten esa lectura de futuro:

i) El tren sale en dos horas $(/ *$ El tren está saliendo en dos horas)

ii) Dijo que el tren salía en dos horas (/ *Dijo que el tren estaba saliendo en dos horas)
} 
Según autores como Mateu y Amadas (1999), lo que el auxiliar be añade es un rasgo locativo que subyace a la interpretación de estas construcciones progresivas en la mayor parte de las lenguas naturales (vid Bybee et al., 1994). Lo cierto es que, independientemente de cómo formalicemos su papel en la construcción, el matiz que to be aporta contribuye a informar sobre cómo el hablante quiere presentar la temporalidad interna del evento, es decir, contribuye a determinar su aspectualidad. Nótese, además, que esta forma analítica es la única que posee el inglés para marcar aspecto imperfectivo en pasado (frente a lenguas como el español donde existe también una forma sintética), y que, por el rasgo de progresión que añade be, de las lecturas básicas asociadas a la imperfectividad, se sigue principalmente la de evento en desarrollo (de ahí que existan otros medios analíticos, como el auxiliar used to, para marcar habitualidad).

Por lo que se refiere al auxiliar have, su contribución no es tanto a la forma de presentar el evento (que se entiende como perfectivo, igual que en el Pretérito, si have es el único auxiliar, y como imperfectivo, si se combina con el auxiliar be) sino a su posicionamiento con respecto a una referencia temporal. ${ }^{6}$ Es decir, to have marca el sintagma verbal como ANTERIOR al momento que se codifica en su morfología: si have está en presente, el evento se presentará como anterior al momento del habla, y si está en pasado como anterior a una referencia ya pasada. ${ }^{7}$ Las peculiaridades del Presente Perfecto se siguen directamente de esta temporalidad compuesta en las formas del perfecto:

\section{9. [have $<$ tiempo gramatical: presente $><$ anterioridad $>]+$ verbo - en $<$ perfectivo $>$}

Puesto que el sintagma verbal perfectivo (i.e. considerado en su totalidad) se sitúa como anterior al presente, la referencia final de la construcción analítica es de pasado. Pero esta lectura no se obtiene de la ordenación directa del evento con respecto al momento del habla, sino de su relación de anterioridad con un auxiliar presente. Así pues, el Presente Perfecto se diferenciará del Pretérito, aspectualmente equivalente a él, precisamente en la obligada conexión del evento con el tiempo del auxiliar: ésta es la base del denominado "requisito de pertinencia" (current relevance), una de las propiedades definitorias del Presente Perfecto y que restringe esta forma a contextos donde existe relación con el presente, sea ésta estrictamente temporal (i.e. el acontecimiento verbal tiene lugar en el marco temporal del acto del habla, como en (10)) o pragmática (i.e. el acontecimiento o sus efectos se sienten como presentes por el hablante, como en el ejemplo (11), o el tema del discurso determina que ese acontecimiento puede repetirse, como en (12)): ${ }^{8}$

\footnotetext{
${ }^{6}$ Quereda (1993) también desvincula al auxiliar have de cualquier implicación aspectual, y denomina la noción gramatical que este auxiliar introduce "fase" (siguiendo a Joos 1964), ya que sirve para conectar dos puntos de referencia temporal, uno de los cuales es necesariamente anterior y pertinente al otro. Nuestro enfoque difiere del suyo en que entendemos que, en las formas del perfecto, el participio pasado sí conlleva también un valor aspectual de perfectividad, lo cual las convierte en funcionalmente equivalentes a las del Pretérito.

7 Significativamente en su teoría sobre el tiempo gramatical, Reichenbach (1947) denomina a las formas analíticas con have, formas "anteriores": Presente/Pasado/Futuro Anterior. Nosotros utilizaremos el término Presente Perfecto, en lugar del más habitual en la tradición hispana de Pretérito Perfecto, porque resulta más coherente con la forma de la construcción.

${ }^{8}$ El requisito de pertinencia como propiedad del Presente Perfecto ya aparece mencionado en Jespersen (1924). Inoue (1979), de quien está tomado el ejemplo (12), analiza esta restricción como posibilidad de
} 
10. She has visited me this morning

11. I have broken my arm

12. Einsten has visited Princetown

La conexión del Presente Perfecto con el tiempo presente del auxiliar have se manifiesta también en una restricción estructural que se da en inglés y algunas lenguas escandinavas: la construcción sólo permite adverbiales que sean compatibles con el presente, es decir, que no señalen un momento definido del pasado: ${ }^{9}$

13. She has left home *a moment ago/*at five

Así pues, las principales características del Presente Perfecto se siguen de su propia naturaleza composicional, que relaciona un evento perfectivo (i.e. completo ya, y por tanto pasado) con el presente morfológico marcado en el auxiliar. La restricción de pertinencia, conjugada con los valores del aspecto léxico del predicado verbal, explica también los usos básicos de esta forma, que tradicionalmente se resumen en tres (vid. Comrie, 1976: 56-61 y Huddleston and Pullum, 2002: 143-147, entre otros):

14. Perfecto de situación persistente: básicamente con predicados atélicos ${ }^{10}$ They have lived here for ten years

15. Perfecto resultativo: básicamente con predicados télicos

She has closed the door

16. Perfecto de pasado reciente: el requisito de pertinencia implica simplemente cercanía temporal

They have just arrived

Obviamente, en las restantes formas del perfecto, i.e. en aquellas donde el auxiliar no tiene morfología presente, ninguna de las restricciones señaladas tienen efecto, y el acontecimiento verbal simplemente se concibe como perfectivo y anterior a una determinada referencia temporal:

17. (They said that) she had left home yesterday / at five

18. To have signed that manifest two years ago was now seen as a sign of weakness

En conclusión: en las formas analíticas el auxiliar be añade un matiz aspectual de progresión, mientras que lo que have aporta es un rasgo de anterioridad, es decir, un rasgo básicamente temporal. Sólo el primero puede por tanto considerarse strictu senso un auxi-

que lo expresado por el predicado sea repetible en el momento presente; la oración en (12) sería, según esto, aceptable sólo si el tema del discurso fuera el comentario de los momentos históricos vividos en Princetown.

9 Esta peculiaridad se ha denominado "Present Perfect Puzzle" y ha sido abordada como una restricción semántica (cf. Dowty, 1979), pragmática (cf. Klein, 1992) e incluso sintáctica (cf. Giorgi y Pianesi, 1997).

10 Vid. García Fernández y Martínez-Atienza (2003) sobre la obligatoriedad del modificador adverbial en esta interpretación del perfecto, que ellos denominan lectura "continuativa". 
liar de aspecto, y este contraste se refleja en determinadas construcciones que analizaremos a continuación.

3. Algunas consecuencias empíricas. Existe un consenso casi generalizado en defender que el orden estructural de las categorías gramaticales de modo, tiempo y aspecto, es precisamente éste: el aspecto domina inmediatamente al sintagma verbal y es dominado por el tiempo, como esquemáticamente se representa en (19): ${ }^{11}$

19. [SModalidad/STiempo [SAspecto [SV ...

Aparte de las implicaciones estructurales y empíricas de esta jerarquía gramatical, se siguen de ella interesantes predicciones sobre el orden en que las nociones asociadas a la morfología verbal serán adquiridas, tanto en la primera lengua como en la segunda: se espera que, en su desarrollo lingüístico, el hablante codifique primero el aspecto y posteriormente el tiempo (y el modo); abundantes trabajos en el marco de la adquisición parecen corroborar este extremo (vid., por ejemplo, Bloom et al. 1980 y Ojea 2001 sobre la adquisición del aspecto en la primera lengua y Bardovi-Harlig, 2000, sobre su adquisición en segundas lenguas).

En lo que se refiere al tema concreto que estamos tratando aquí, si el tiempo gramatical domina al aspecto, se predice que en las formas analíticas en las que coexisten los dos auxiliares el que informa principalmente del tiempo dominará al que informa principalmente del aspecto verbal. Y efectivamente, el orden de los dos auxiliares es necesariamente have+be:

20. He has been working in his thesis for three years

*He was having worked in his thesis for three years

Nótese que en la forma compuesta con los dos auxiliares (las denominadas formas del perfecto continuo) have marca como anterior una forma imperfectiva, tal y como puede comprobarse en los contrastes de (21) -(23), tomados de Quirk et al. (1972: 97), Quirk et al. (1985: 211) y Huddleston y Pullum (2000: 165), respectivamente:

21. a. Who's eaten my dinner? (implicación: no queda nada)

b. Who's been eating my dinner? (implicación: queda algo todavía)

22. a. *I have written a novel, but I haven't finished it

b. I've been writing a novel, but I haven't finished it

23. a. He has learnt to swim (implicación: ahora sabe nadar)

b. He's been learning to swim (implicación: todavía no sabe nadar)

Lo que estos ejemplos vienen a demostrar es que, tal y como señalamos en (4) y (9), el aspecto gramatical [ \pm perfectivo] en las formas analíticas viene determinado por la forma del verbo principal, no por el auxiliar. El participio presente es una forma intrínsicamente

\footnotetext{
${ }^{11}$ No entramos a discutir si SMod y ST son compatibles o se excluyen, o si habría que distinguir entre una proyección de SAsp para el aspecto léxico y otra para el gramatical. Vid. Cinque (1999) para una justificación exhaustiva de las distintas categorías gramaticales y su ordenación.
} 
[-perfectiva], y el auxiliar be sólo contribuye añadiéndole un rasgo de progresión que no es inherente a la noción de imperfectividad. Por su parte, el participio pasado es una forma intrínsicamente [+perfectiva], y cuando se combina con have el auxiliar lo sitúa en el eje temporal como anterior a una determinada referencia temporal, pero no contribuye con ningún rasgo aspectual extra. Hasta tal punto esto es así que cuando el complemento de have es una forma [-perfectiva] con be, la forma compuesta mantiene esa lectura imperfectiva, tal y como se observa en las implicaciones de $21 \mathrm{~b}-23 \mathrm{~b}$ que claramente reflejan la denominada "paradoja imperfectiva".

Volviendo a las construcciones de perfecto no progresivo, si el único matiz que have añade es de temporalidad, es predecible que en aquellos casos en que la expresión de anterioridad no sea estrictamente necesaria las formas perfectivas simples y compuestas se neutralicen; tal situación es frecuente entre el Pretérito y el Pasado Perfecto:

\section{They began to pack after the children had come / came back from school}

Otro tanto ocurre con el Presente Perfecto y el Pretérito simple. Como ya señalamos, ambas formas son equivalentes en lo que a su temporalidad se refiere, siendo la única diferencia importante entre ellas el hecho de que la referencia temporal la marque directamente el momento del habla o un auxiliar en el tiempo gramatical presente. No es extraño, por tanto, que en lenguas donde restricciones estructurales como la que condiciona la gramaticalidad de (13) no tienen efecto (i.e. en las lenguas románicas y la mayor parte de las germánicas), las dos formas puedan alternar con bastante libertad. ${ }^{12}$ Incluso en inglés, si no aparece ningún adverbial incompatible con la marca de presente de have, es posible la neutralización de las dos formas:

25. I may have left my umbrella at the pub

a) It is possible that I left my umbrella at the pub

b) It is possible that I have left my umbrella at the pub

Queda claro, por tanto, que have no es un auxiliar de aspecto gramatical, si por tal se entiende un elemento que aporta matices específicos que contribuyen a caracterizar la temporalidad interna del predicado verbal.

Queremos, por último, señalar un hecho que viene a abundar en la diferente naturaleza de be y have como auxiliares, ya que corrobora que sólo el primero añade información de tipo aspectual. Es bien sabido que los verbos modales tienen dos lecturas principales: la epistémica y la deóntica (vid. Palmer, 1986). En el primer caso, se evalúa la actitud del hablante sobre el valor de verdad de la proposición ((im)posible, (im)probable, (in)necesaria...), sea ésta presente o pasada; dado que los modales seleccionan infinitivo sin to, será el infinitivo perfecto (i.e. con have) el que marque la proposición como pasada:

\footnotetext{
12 Piénsese, por ejemplo, en el caso concreto del español: tanto en ciertas variedades peninsulares (e.g. Galicia, Asturias, León) como hispanoamericanas (e.g. Méjico, Colombia, Argentina...), el Pretérito simple normalmente adopta usos y lecturas asociadas con los valores del Presente Perfecto (vid. Cartagena, 1999).
} 
26. a. He may sleep here tonight

b. He may have slept here last night

27. a. You must be very patient now

b. You must have been very patient then

En cuanto a la modalidad deóntica, ésta expresa la implicación del hablante sobre el acontecimiento verbal (en términos de imposición, deseo, permiso....). Las oraciones (26a) y (27a) son ambiguas y pueden expresar también modalidad deóntica, con el sentido de permiso y de imposición por parte del hablante respectivamente; esta situación no se da en (26b) y (27b) dada la incompatibilidad entre la modalidad deóntica presente y la proposición pasada (i.e. no se puede expresar un permiso o un mandato en presente sobre algo ya pasado). ${ }^{13}$

Así pues, el auxiliar have sólo determina el tiempo de la proposición, con lo que eso conlleva de posible incompatibilidad semántica entre ésta y determinadas lecturas del verbo modal. En cuanto a be, también sirve para activar ciertas lecturas del modal, al marcar el infinitivo desnudo de la proposición como imperfectivo y progresivo, es decir, al presentar el acontecimiento como aspectualmente no delimitado. Pensemos en ejemplos como (28) o (29):

28. a. He may play the piano (principalmente lectura deóntica)

b. He may have played the piano (sólo lectura epistémica)

c. He may be playing the piano (principalmente lectura epistémica)

29. a. He must write a letter to his mother (principalmente lectura deóntica)

b. He must have written a letter to his mother (sólo lectura epistémica)

c. He must be writing a letter to his mother (principalmente lectura epistémica)

Cuando el predicado de la proposición complemento del modal no está en pasado (como en 28b y 29b), la lectura que se sigue es principalmente de tipo deóntico (28a y 29a); ahora bien, se puede obtener también una lectura epistémica si este predicado es aspectualmente imperfectivo, es decir, si aparece con el auxiliar be (28c y 29c). Sin intentar plantear una explicación exhaustiva del porqué de esta asociación, intuitivamente parece relacionarse con el hecho de que la modalidad deóntica implica algún tipo de control sobre el evento verbal por parte del hablante (mandato, concesión de permiso...) y esta implicación se sigue más directamente si se presenta el evento en su totalidad, es decir, como delimitado cuando su significado es susceptible de delimitación. ${ }^{14}$

Así pues, los auxiliares have y be se combinan con el verbo modal para activar una determinada modalidad, en concreto la epistémica, pero, tal y como hemos venido mante-

\footnotetext{
${ }_{13}$ Sí sería posible, no obstante, la expresión de modalidad deóntica en el pasado con una proposición pasada, siguiéndose entonces la implicación de que los hechos finalmente no tuvieron lugar: He should have played the piano (implicación: pero no lo hizo).

${ }^{14}$ Obviamente hay otros factores semántico-discursivos que potencian una lectura modal frente a otras. Por ejemplo, la lectura deóntica se obtiene más fácilmente si el sujeto de la proposición es el oyente (i.e. la segunda persona, you), es decir, el argumento que funciona como agente del predicado sobre el que se quiere influir. Nótese, no obstante, que los casos en que el modal se interpreta ambiguamente incluso si el sujeto gramatical es you (e.g. 26a y 27a) suelen ir ligados a predicados atélicos, donde la delimitación del evento es semánticamente imposible.
} 
niendo, mientras que have impide la modalidad deóntica al marcar la proposición como pasada (información temporal), be potencia la modalidad epistémica al marcar el evanto como no delimitado (información aspectual).

\section{REFERENCIAS BIBLIOGRÁFICAS}

AArTs, B. 1997. English Syntax and Argumentation. London: McMillan Press.

BARDOVI-Harlig, K. 2000. Tense and aspect in second language acquisition: form, meaning and use. Malden, MA: Blackwell.

BloOM, L., K. LifTER y J. Hafitz. 1989. "Semantics of verbs and the development of verb inflection in child language". Language 56.2. 386-412.

Bybee, J. L. 1994. The Evolution of Grammar: Tense, Aspect and Modality in the Languages of the World. Chicago: University of Chicago Press.

Cartagena, N. 1999. "Los tiempos compuestos". Gramática descriptiva de la lengua española, ed. I. Bosque y V. Demonte. Madrid: Espasa, 2935-2975.

Cinque, G. 1999. Adverbs and Functional Heads. Oxford: Oxford University Press.

Comrie, B. 1976. Aspect. Cambridge, UK.: Cambridge University Press.

DowTy, D. R. 1979. Word Meaning and Montague Grammar. Dordrecht: Reidel.

García Fernández, L. y M. Martínez Atienza. 2003. "La expresión de los eventos inconclusos en español”. Revista Española de Lingüística 33, 1: 29-67.

Giorgi, A. y F. Pianesi. 1997. Tense and Aspect. From Semantics to Morphosyntax. Oxford: Oxford University Press.

Huddleston, R. 1984. Introduction to the Grammar of English. Cambridge, UK: Cambridge University Press.

Huddleston, R. y G. K. Pullum. 2002. The Cambridge Grammar of English. Cambridge, UK: Cambridge University Press.

INOUE, K.. 1979. “An analysis of the English present perfect”. Linguistics 17. 561-589.

Joos, M. 1964. The English Verb: Forms and Meanings. Madison: University of Wisconsin Press.

Jespersen, O. 1924. The Philosophy of Grammar. London: Allen \& Unwin.

KLEIN, W. 1992. "The present perfect puzzle”. Language 68. 525-552.

Mateu Fontanals, J. y L. Amadas Simon. 1999. "Extended argument structure: progressive as unacussative". Catalan Working Papers in Linguistics 7. 159-174

NAPOLI, D. J. 1993. Syntax. Oxford: Oxford University Press.

OJEA, A. 2001. "El desarrollo sintáctico en la adquisición de la primera lengua: análisis de la etapa telegráfica de una sujeto monolingüe de español”. Revista Española de Lingüística 31, 2. 413-430. 
- 2003. "Tense and aspect in English and Spanish past forms". Southwest Journal of Linguistics 22.2.

Palmer, F. R. 1986. Mood and Modality. Cambridge: Cambridge University Press.

Quereda Rodriguez-Navarro, L. 1993. A Morphosyntactic Study of the English Verb Phrase, Granada: Servicio de Publicaciones de la Universidad de Granada.

Quirk, R., S. Greenbaum, G. LeEch, y J. Svartvik. 1972. A Grammar of Contemporary English. London: Longman.

1985. A Comprehensive Grammar of the English Language. London: Longman.

RAdFord, A. 1997. Syntactic Theory and the Structure of English. Cambridge: Cambridge University Press.

ReICHEnBACH, H. 1947: Elements of Symbolic Logic. New York: Free Press.

Sмiтн, C. S. 1991. The Parameter of Aspect. Dordrecht: Kluwer.

VendLer, Z. 1957. "Verbs and times”. The Philosophical Review 66. 143-160. 